\title{
Inflammatory response of macrophages and trophoblasts investigated using structured illumination microscopy and quantitative phase microscopy
}

\author{
Rajwinder Singh ${ }^{1}$, Deanna L. Wolfson ${ }^{1}$, Vishesh Dubey ${ }^{1,2}$, Azeem Ahmad ${ }^{1,2}$, Ganesh Acharya ${ }^{3,4}$, D. S. Mehta ${ }^{2}$, Purusotam \\ Basnet $^{3, \#}$, Balpreet S. Ahluwalia ${ }^{1, *}$ \\ 1. Department of Physics and Technology, UiT-The Arctic University of Norway, 9037 Tromsø, Norway \\ 2. Department of Physics, Indian Institute of Technology, 110016 New Delhi, India \\ 3. Department of Clinical Medicine, UiT-The Arctic University of Norway, 9037 Tromsø, Norway \\ 4. Department of Clinical Science Intervention and Technology, Karolinska Institutet, 14186 Stockholm, Sweden
}

\#purusotam.basnet@uit.no *balpreet.singh.ahluwalia@uit.no

Introduction: The super-resolution capability of structured illumination microscopy (SIM) (100 nm) enables 3D imaging of mitochondria, and label-free quantitative phase microscopy (QPM) provides nanoscale quantitative phase values relating to cellular thickness and the refractive index of cellular content.

Methods: We examined morphological changes in response to LPS and TNF- $\alpha$ (agents which are known to cause inflammation) challenge on live RAW264.7 (macrophage) and HTR-8/SVneo (trophoblast) cell lines using SIM and QPM, and quantitatively measured nitric oxide (NO) production.

Results: SIM imaging showed changes in the morphology of mitochondria (Fig.1) and plasma membrane (Fig.2) in approximately $50-60 \%$ of macrophages following LPS challenge $(1 \mu \mathrm{g} / \mathrm{ml}$ for $24 \mathrm{~h})$, but no detectable changes in mitochondria or plasma membrane were observed after TNF- $\alpha$ challenge ( $1 \mathrm{ng} / \mathrm{ml}$ for $24 \mathrm{~h}$ ). Mitochondrial and plasma membrane morphology appeared unaffected in trophoblasts following either LPS or TNF- $\alpha$ challenge under similar conditions. LPS-challenged macrophages produced approximately 22 fold more NO as compared to controls, whereas no significant increase was seen after TNF- $\alpha$ challenge. QPM revealed that the phase value decreased by approximately $18 \%$ in LPS-challenged macrophages as compared to controls. In contrast, no notable changes in the phase value or NO production were observed in trophoblasts with either LPS or TNF- $\alpha$ challenge.

Conclusion: Our results suggest that the different cells which are responsible for the mother-fetus cross-talk, especially macrophages and trophoblasts, respond differently to various inflammatory agents. High-resolution optical microscopy is shown to be a live-cell friendly, useful tool to evaluate sub-cellular mechanisms associated with inflammation complications during pregnancy.
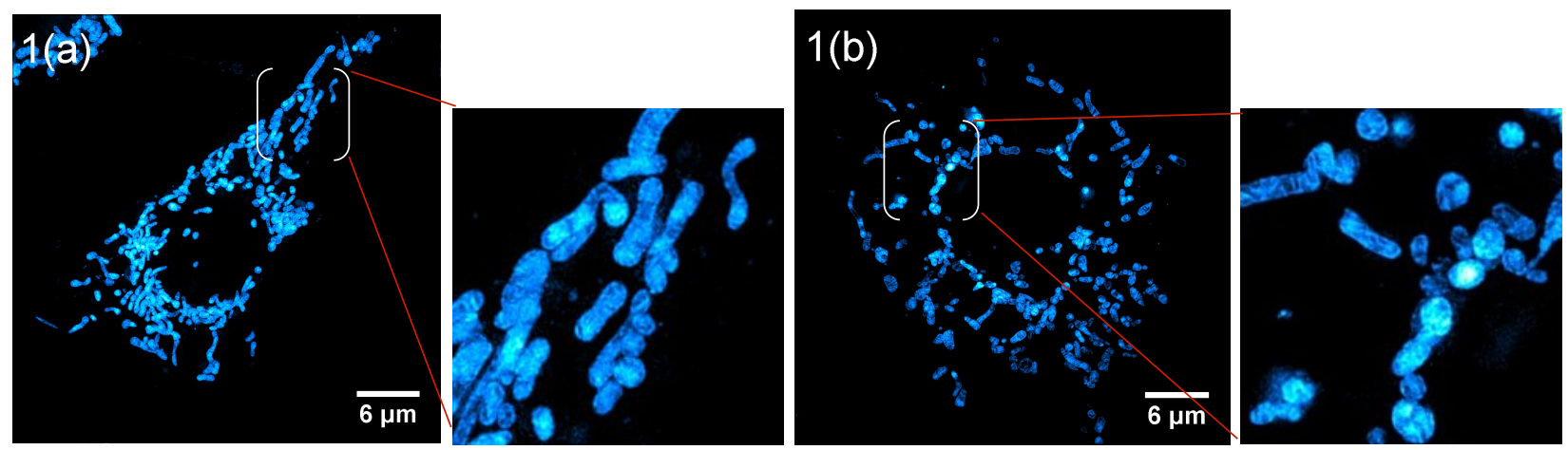

Fig. 1. In controls (a), mitochondria appeared longer and more uniform while in LPS-challenged macrophages (b) they were smaller and rounded in shape.
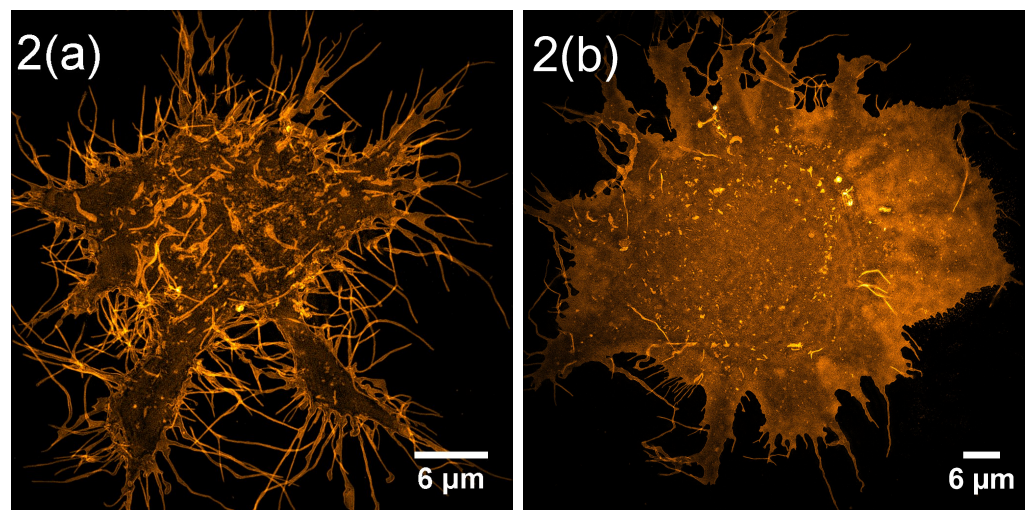

Fig. 2. Compared to controls (a), LPS-challenged macrophages (b) have larger footprints and fewer protruding extensions. 